\title{
Liposomes as carriers: not as innocent as one would like
}

\author{
Rolf Gebhardt $^{1} \cdot$ Madlen Matz-Soja $^{1}$
}

Received: 22 July 2015 / Accepted: 27 July 2015 / Published online: 6 August 2015

(C) Springer-Verlag Berlin Heidelberg 2015

In the postgenomic area, elucidation of the function and regulation of unknown genes is a major challenge of biological research. Oligonucleotides and siRNAs are important tools aiding in this endeavour (de Fougerolles et al. 2007) and, due to their remarkable specificity covering a wide range of applications, have considerably advanced our knowledge of physiological and pathological functions of the respective gene products (Raouane et al. 2012). The increasing number of potential therapeutic targets resulting from such studies makes these tools likewise attractive for inhibiting those target proteins that are not easily accessible and responsive to conventional small molecular weight or protein drugs (Xu and Anchordoquy 2011; Bartneck et al. 2014; Lorenzer et al. 2015).

Efficient cellular delivery of these molecules and specific targeting of selected cell types are important goals that drive the development of various types of delivery systems, such as liposomes, micelles, nanoparticles, lipidated macromolecules, and polyplexes (Wu and McMillan 2009; Raouane et al. 2012; Navarro et al. 2015). Liposomes seem to be an almost ideal carrier system, since their morphology shows a high degree of similarity to cellular membranes and because they are able to incorporate various substances (Bozzuto and Molinari 2015). Further, they can be modified at the surface or equipped with additional molecules favouring fusion with or endocytosis by specific types of cells. These biological features and technological advantages have made liposomes one of the most versatile carrier

Rolf Gebhardt

rgebhardt@medizin.uni-leipzig.de

1 Faculty of Medicine, Institute of Biochemistry, University of Leipzig, Johannisallee 30, 04103 Leipzig, Germany systems available that is valued for its simple applicability, both in vitro and in vivo (Bozzuto and Molinari 2015).

Among the few drawbacks of liposomes, cytotoxicity and immunomodulation have attracted most attention and many studies have been performed to minimise such adverse effects (Battaglia and Gallarate 2012; Peer 2012). At the sub-toxic level, however, possible interactions with cellular metabolism and function were largely ignored, because most components are identical to cellular ingredients or are easily degradable by lysosomal and other cellular enzymes (Bozzuto and Molinari 2015). That this view may be a little bit too simple has been brought to our attention by a thorough comparative study of the influence of three lipid-based transfection agents on hepatocytes focusing on various side effects on cellular metabolism presented in this issue of Archives of Toxicology (Böttger et al. 2015). While this study confirmed the high efficiency of the tested transfection agents in silencing transcription of target genes by respective siRNAs together with a low cytotoxicity for the hepatocytes (Bakhshandeh et al. 2012; Gao et al. 2012), it demonstrated considerable effects on different aspects of lipid and amino acid metabolism of these cells (Böttger et al. 2015). Using highly sophisticated shotgun lipidomics analysis, Böttger et al. (2015) showed that all tested transfection agents altered the hepatocellular accumulation of triglycerides (two up and one down). The changes were most pronounced in triglycerides containing moderately unsaturated fatty acids. Furthermore, the expression levels of certain enzymes and regulatory factors associated with lipid metabolism were affected to a variable degree. Interestingly, no significant changes could be observed with respect to most phospholipid species, indicating that the balance of membrane lipids in hepatocytes is not disturbed by the presence of the liposomes. Analysis of the exo-metabolome revealed that the changes in the 
transfected cells are not restricted to lipid metabolism, but also concerned the metabolism of amino acids and alphaketo acids such as pyruvate (Böttger et al. 2015). These changes indicate an influence of the transfection agents on mitochondrial functions and energy metabolism.

The findings of Böttger et al. (2015) clearly demonstrate that exposing hepatocytes to liposome- and/or lipid-based transfection agents can lead to considerable alterations of metabolic features of these cells. These observations raise a number of questions: (A) Are these effects hepatocyte-specific? Though the molecular mechanisms of these changes were only peripherally addressed by Böttger et al. (2015), there seems no doubt that cellular sensing of lipid components may play a role. Sensing of such components similar to hepatocytes is found also in other cell types, e.g. neurons, endocytes, or pancreatic cells (Pardridge 2007; Moullé et al. 2014; Rütti et al. 2014; Hirasawa 2015) and, thus, may provide the basis for similar changes in many other cells. (B) Can these findings be generalised with respect to the molecular composition of the delivery system? The pronounced differences between the tested transfection agents in affecting metabolic and regulatory features show that the individual molecular composition is of utmost importance. However, this may hold also for lipid-modulated structures used in other delivery systems (Zhang et al. 2013; Raouane et al. 2012; Navarro et al. 2015) and calls for careful analysis of cellular changes provoked by these compounds. (C) What are the consequences for RNA interference studies and other applications? As Böttger et al. (2015) have pointed out, their findings add another type of uncertainty to siRNA-mediated gene silencing. Researchers not only have to look for off-target effects of the siRNA used, but also need to rule out by rigorous testing that their results are not biased by carrier-induced disturbance of regulatory networks for transcription. When lipid-based carriers are used for delivery of therapeutic oligonucleotides or other drugs in vivo, the importance for such testing may even increase, since even moderate disturbance of metabolism in certain cell types or systemically may lead to intolerable side effects of the therapeutic approach. In any case, we need to realise that targeting cells with liposome-like structures is not just a matter of life (survival) or death (cytotoxicity), but of a wide spectrum of cellular responses which may be tolerable or not.
Acknowledgments The authors would like to acknowledge the support by the BMBF Virtual Liver Program.

\section{References}

Bakhshandeh B, Soleimani M, Hafizi M et al (2012) A comparative study on nonviral genetic modifications in cord blood and bone marrow mesenchymal stem cells. Cytotechnology 64:523-540

Bartneck M, Warzecha KT, Tacke F (2014) Therapeutic targeting of liver inflammation and fibrosis by nanomedicine. Hepatobiliary Surg Nutr 3:364-376

Battaglia L, Gallarate M (2012) Lipid nanoparticles: state of the art, new preparation methods and challenges in drug delivery. Expert Opin Drug Deliv 9:497-508

Böttger J, Arnold K, Thiel C et al (2015) RNAi in murine hepatocytes: the agony of choice-a study of the influence of lipidbased transfection reagents on hepatocyte metabolism. Arch Toxicol. doi:10.1007/s00204-015-1571-0

Bozzuto G, Molinari A (2015) Liposomes as nanomedical devices. Int J Nanomed 10:975-999

de Fougerolles A, Vornlocher H, Maraganore J et al (2007) Interfering with disease: a progress report on siRNA-based therapeutics. Nat Rev Drug Discov 6:443-453

Gao W, Lai James C K, Leung SW (2012) Functional enhancement of chitosan and nanoparticles in cell culture, tissue engineering, and pharmaceutical applications. Front Physiol 3:321

Hirasawa A (2015) Free fatty acid receptor family: a new therapeutic target for metabolic diseases. Yakugaku Zasshi 135:769-777

Lorenzer C, Dirin M, Winkler A et al (2015) Going beyond the liver: progress and challenges of targeted delivery of siRNA therapeutics. J Control Release 203:1-15

Moullé V, Picard A, Le Foll C et al (2014) Lipid sensing in the brain and regulation of energy balance. Diabetes Metab 40:29-33

Navarro G, Pan J, Torchilin VP (2015) Micelle-like nanoparticles as carriers for DNA and siRNA. Mol Pharm 12:301-313

Pardridge WM (2007) shRNA and siRNA delivery to the brain. Adv Drug Deliv Rev 59:141-152

Peer D (2012) Immunotoxicity derived from manipulating leukocytes with lipid-based nanoparticles. Adv Drug Deliv Rev 64:1738-1748

Raouane M, Desmaele D, Urbinati G et al (2012) Lipid conjugated oligonucleotides: a useful strategy for delivery. Bioconjug Chem 23:1091-1104

Rütti S, Arous C, Nica AC et al (2014) Expression, phosphorylation and function of the Rab-GTPase activating protein TBC1D1 in pancreatic beta-cells. FEBS Lett 588:15-20

Wu SY, McMillan NAJ (2009) Lipidic systems for in vivo siRNA delivery. AAPS J 11:639-652

Xu L, Anchordoquy T (2011) Drug delivery trends in clinical trials and translational medicine: challenges and opportunities in the delivery of nucleic acid-based therapeutics. J Pharm Sci 100:38-52

Zhang Y, Wang Z, Gemeinhart RA (2013) Progress in microRNA delivery. J Control Release 172:962-974 